\title{
Pathology of Striped Dolphins (Stenella coeruleoalba) Infected with Brucella ceti
}

\author{
R. González-Barrientos ${ }^{*}$, J.-A. Morales ${ }^{*}$, G. Hernández-Mora ${ }^{\dagger}$, \\ E. Barquero-Galvo ${ }^{\dagger}$, C. Guzmán-Verri ${ }^{\dagger}$, E. Chaves-Olarte ${ }^{\dagger,}$ \\ and E. Moreno ${ }^{\dagger}, \S$ \\ * Cátedra de Patología, Escuela de Medicina Veterinaria, ${ }^{\dagger}$ Programa de Investigación en Enfermedades Tropicales, Escuela de \\ Medicina Veterinaria, Universidad Nacional, Heredia, ${ }^{\star}$ Centro de Investigación en Enfermedades Tropicales, Facultad de \\ Microbiología and ${ }^{\S}$ Instituto Clodomiro Picado, Universidad de Costa Rica, San José, Costa Rica
}

\begin{abstract}
Summary
Seventeen striped dolphins (Stenella coeruleoalba) displaying swimming disorders compatible with neurological syndromes were investigated for Brucella infection. Sixteen dolphins had meningoencephalomyelitis. Serum antibody against Brucella antigen was detected in all 14 animals tested and Brucella ceti was isolated from eight out of nine animals. Brucella antigen was detected in the brain by immunofluorescence, but not by immunohistochemical labelling. By contrast, Brucella antigen was demonstrated by immunohistochemistry in the trophoblast of animals with severe placentitis and in the mitral valve of animals with myocarditis. The microscopical lesions observed in the tissues of the infected dolphins were similar to those of chronic brucellosis in man. The severity of brucellosis in $S$. coeruleoalba indicates that this dolphin species is highly susceptible to infection by $B$. ceti.
\end{abstract}

(C) 2009 Elsevier Ltd. All rights reserved.

Keywords: Brucella; Brucella ceti; endocarditis; neurobrucellosis; placentitis; Stenella coeruleoalba; striped dolphin

Members of the genus Brucella are intracellular pathogens of marine and terrestrial mammals, including man (Meador et al., 1989; Ewalt et al., 1994; Foster et al., 2002; Pappas et al., 2005; Groussaud et al., 2007). The infection may pass unnoticed in non-pregnant natural hosts and in ill animals in which specific diagnostic procedures are not performed (BarqueroCalvo et al., 2007). However, in gravid females the infection generally causes abortion and in males epididymitis and orchitis. In contrast, in secondary accidental hosts such as man, the infection commonly causes a severe and obvious illness with a broad spectrum of symptoms that may become grave if not treated (Pappas et al., 2005).

Brucellosis in cetaceans is caused by Brucella ceti, a species that is predominant in dolphins and whales (Groussaud et al., 2007). The B. ceti group, which may comprise at least two distinct strains (dolphin and

Correspondence to: E. Moreno (e-mail: emoreno@racsa.co.cr). porpoise types), is phenotypically similar to smooth Brucella abortus and Brucella melitensis, possessing the same surface antigens that are commonly used for the serological diagnosis of brucellosis in infected cattle (Baucheron et al., 2002; Groussaud et al., 2007). Moreover, marine Brucella strains have been described causing lesions in both cetaceans and man (Ewalt et al., 1994; Brew et al., 1999; Miller et al., 1999; González et al., 2002; Sohn et al., 2003; McDonald et al., 2006; Hernández-Mora et al., 2008) and experimental infection with these strains may induce seroconversion and abortion in cattle (Rhyan et al., 2001).

The isolation and characterization of $B$. ceti strains from the cerebrospinal fluid of striped dolphins (Stenella coeruleoalba) stranded on the Pacific shoreline of Costa Rica has been described previously (Hernández-Mora et al., 2008). The present report extends these findings and describes pathological lesions in 17 affected striped dolphins and one fetus. 
Between 2001 and 2009, 17 striped dolphins were stranded on the Pacific shorelines of Costa Rica (Table 1). All of these animals displayed swimming disorders compatible with neurological syndromes before death. Necropsy examinations were performed by the Pathology Unit of the Veterinary School at the National University, Costa Rica. Blood samples were taken from the arterial plexus of 14 dolphins and serum was separated from the clot by centrifugation. Serum samples were tested for the presence of antibody to Brucella by the Rose Bengal test and indirect enzyme-linked immunosorbent assay (ELISA) (Hernández-Mora et al., 2009). All 14 animals were seropositive (Table 1).

During the gross necropsy examination samples were collected from a range of organs and tissues and fixed in 10\% neutral buffered formalin. These were subsequently embedded in paraffin wax and sections were prepared for staining by haematoxylin and eosin (Kiernan, 2003). Smears taken during the gross examination were stained by the Wright-Giemsa method. Bacterial isolation and characterization was performed as described by Hernández-Mora et al. (2008). B. ceti was isolated from the brain and tissues of eight out of nine dolphins and the fetus (Table 1). Bacterial isolation was not attempted in the seven dolphins collected before 2005 .

The major gross and microscopical findings are presented in Figs. 1 and 2. Many of the general pathological findings were not related to brucellosis, but the changes detected in the central nervous system, female reproductive system and heart were associated with Brucella infection. The most significant findings in the brain and meninges have been described previously (González et al., 2002; Muñoz et al., 2006; Hernández-Mora et al., 2008). Sixteen of the 17 animals had meningoencephalomyelitis with little or no involvement of the neural tissue. One juvenile male had hydrocephalus involving the lateral ventricles (Fig. 3A). Hyperaemia of the meninges and brain

Table 1

Meningoencephalomyelitis, detection of anti-Brucella antibodies and $B$. cet $i$ isolation in 17 stranded S. coeruleoalba dolphins

\begin{tabular}{lcccc}
\hline Sex & Age & Meningoencephalomyelitis & $\begin{array}{c}\text { Positive } \\
\text { serology }\end{array}$ & $\begin{array}{c}\text { B. ceti } \\
\text { isolation }\end{array}$ \\
\hline Female & Adult & $6 / 6$ & $5 / 5$ & $4 / 4$ \\
Female & Juvenile & $2 / 2$ & $1 / 1$ & $1 / 1$ \\
Female & Calf & $0 / 1$ & $1 / 1$ & $0 / 0$ \\
Male & Adult & $2 / 2$ & $2 / 2$ & $1 / 1$ \\
Male & Juvenile & $6 / 6$ & $5 / 5$ & $2 / 3$ \\
Total & & $16 / 17$ & $14 / 14$ & $8 / 9$ \\
\hline
\end{tabular}

*Rose Bengal test and indirect ELISA. and cloudiness of the cerebrospinal fluid with increased cellularity was noted in 16 cases. Widespread periventricular encephalitis involving mononuclear cell infiltration was principally found around the third and fourth ventricles. Non-suppurative meningitis affected the spinal cord, medulla oblongata and cerebellum, but this lesion was milder in the meninges overlying the cerebral cortices. In most cases there was perivascular mononuclear infiltration of the white and grey matter of the cerebrum, cerebellum and brainstem, as previously reported (HernándezMora et al., 2008). Moderate to severe non-suppurative choroiditis and major loss of ependyma was also present. Plasma cells, small lymphocytes and macrophages dominated the cellular infiltrates. One juvenile male displaying meningoencephalomyelitis also had fibrinopurulent osteoarthritis with severe infiltration of the synovial fluid by macrophages and neutrophils affecting the right scapulohumeral joint. This change has been described previously in cetaceans with brucellosis (Dagleish et al., 2007). Nine dolphins with meningoencephalomyelitis (six with positive serology and four with positive $B$. ceti cultures) also had non-suppurative interstitial pneumonia and five others displayed periportal lymphocytic hepatitis.

Detection of Brucella antigen in smears was undertaken by immunofluorescence (Hernández-Mora et al., 2008). Detection of Brucella antigen in tissue was undertaken by immunohistochemistry (IHC) by use of the streptavidin-biotin-horseradish peroxidase (HRP) method with rabbit anti-Brucella lipopolysaccharide antibody as primary reagent (Boenish 2001; Hernández-Mora et al., 2008, 2009). The presence of morbillivirus antigen in the brain was explored by IHC (Domingo et al., 1992) with sections of brain from a dolphin with known morbillivirus encephalitis as positive control. The presence of helminths was estimated by macroscopic and microscopic examination of tissues, and of Toxoplasma parasite infections by serology, or histological examination (O'Shea et al., 1991; Dubey et al., 2007).

Brain impressions and smears of cerebrospinal fluid were positive for Brucella by immunofluorescence in the nine animals tested (data not shown). B. ceti was cultured from eight of these animals (Table 1). In contrast, immunohistochemical examination of brain, medulla, cerebellum and spinal cord tissues failed to demonstrate Brucella or morbillivirus antigen in the 17 dolphins tested.

The only pregnant dolphin had severe placentitis with multiple necrotic foci and a dead fetus. The dolphin was estimated to have been in the seventh month of gestation (Hernández-Mora et al., 2008). Microscopical examination of the placenta confirmed severe and widespread necrosis (Fig. 3B) with abundant 


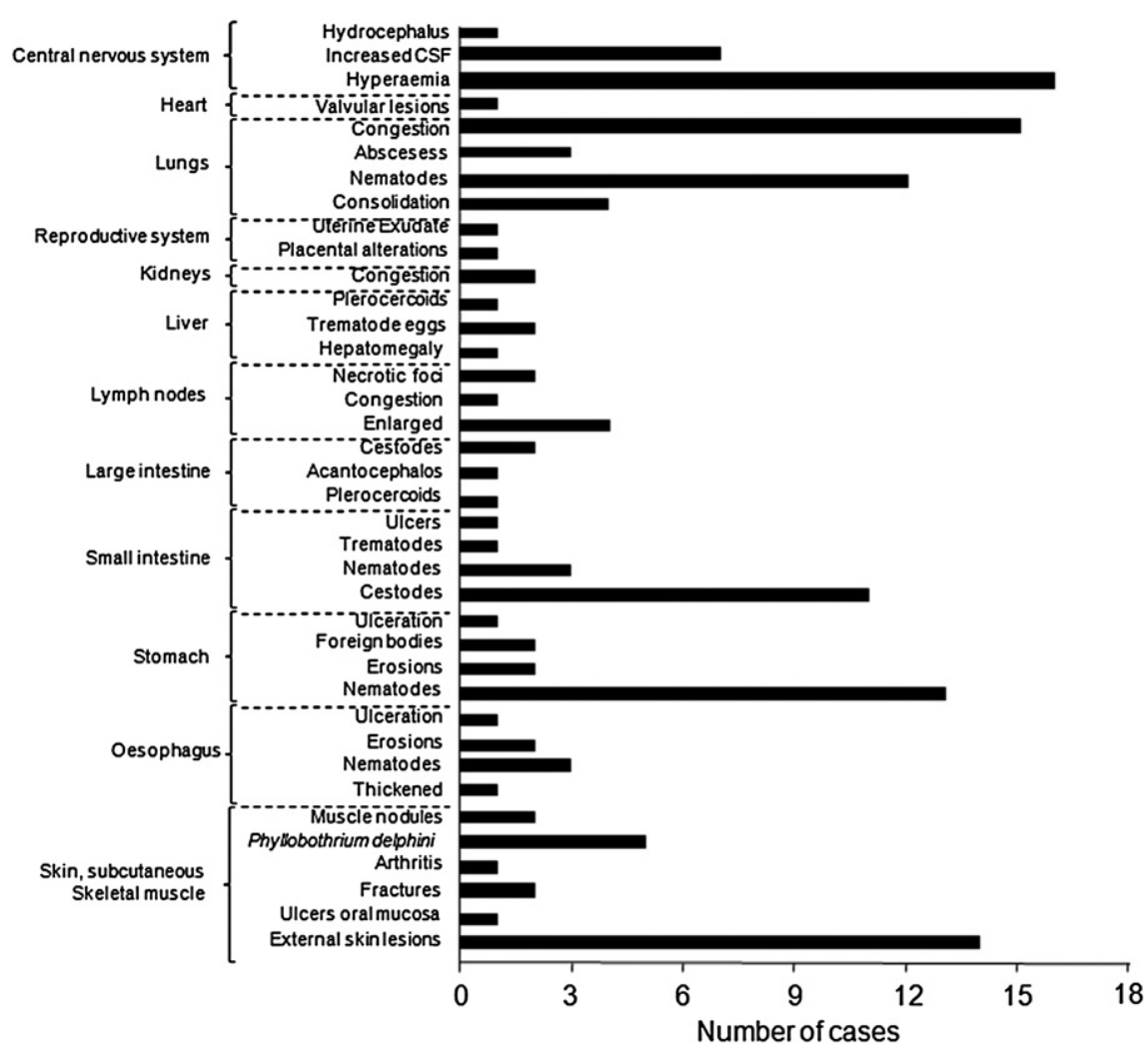

Fig. 1. Gross pathological findings detected in 17 S. coeruleoalba dolphins.

mixed mononuclear and polymorphonuclear infiltration of the trophoblast (Fig. 3G). Brucella antigen was detected by IHC within the inflammatory infiltrate as well as in some chorionic cells in these necrotic regions, the intensity of labelling consistent with the presence of large numbers of bacteria (Fig. 3D). Despite the placental lesions, no significant pathological changes were detected in the fetus.
One adult female had severe endocarditis with thickening and a prominent vegetative nodule of the mitral valve (Fig. 3E). The endocarditis was characterized by the presence of fibrin adjacent to the surface of the mitral valve, with a predominantly non-suppurative infiltration of lymphocytes, macrophages, plasma cells and multinucleate giant cells (Fig. 3F-H). Some scattered necrotic areas with

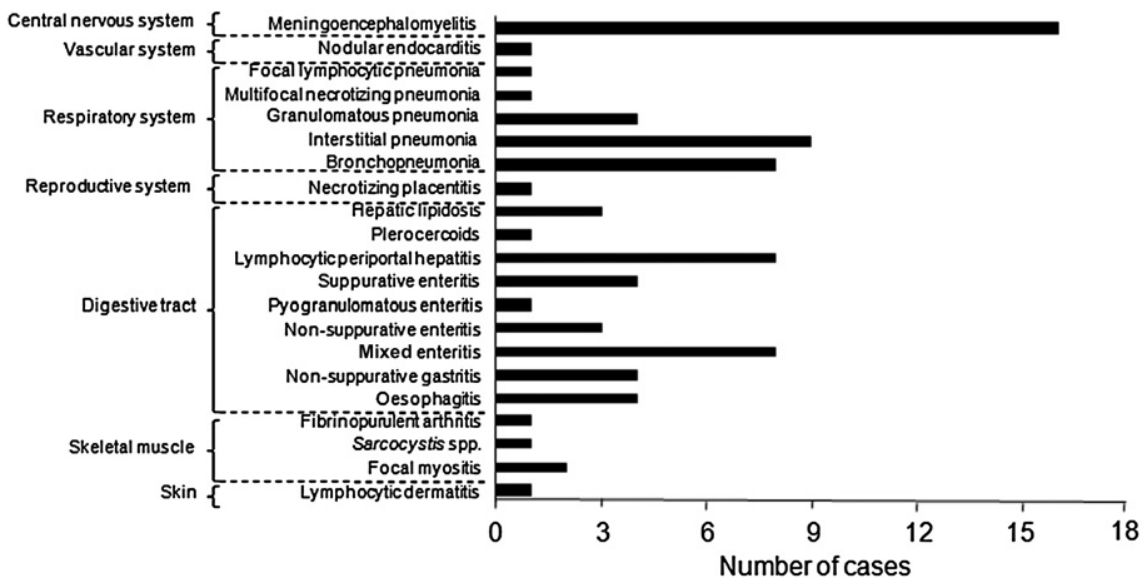

Fig. 2. Microscopical findings detected in 17 S. coeruleoalba dolphins. 


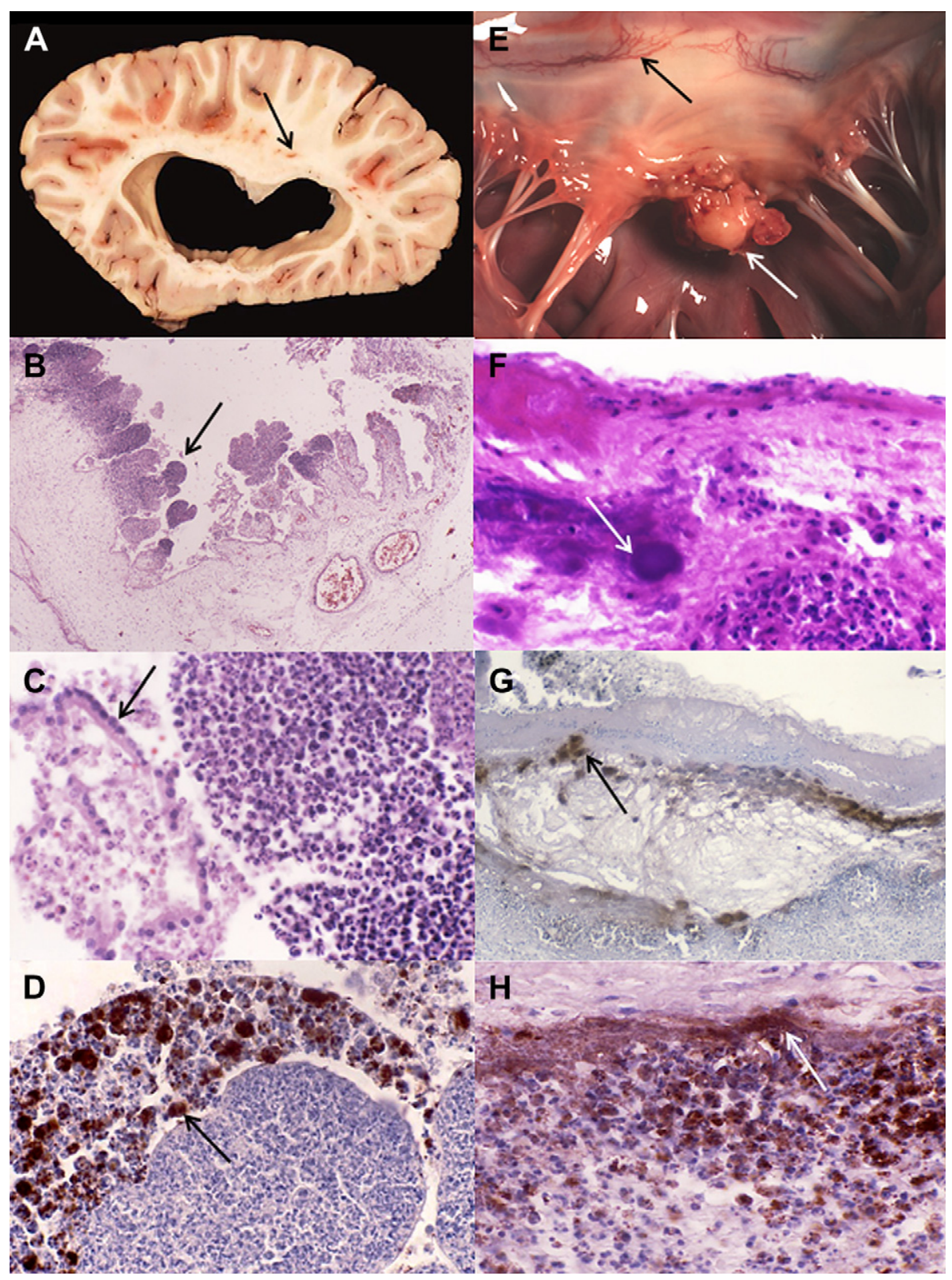

Fig. 3. Pathological findings in B. ceti-infected S. coeruleoalba dolphins. (A) Coronal transverse section of the lateral caudal region of the left hemisphere of the brain. There is internal hydrocephalus with enlargement of the lateral ventricles due to cerebrospinal fluid accumulation secondary to inflammation surrounding the ventricular system. Arrow indicates a hyperaemic blood vessel. (B) Necrotizing placentitis showing marked infiltration of inflammatory cells into the fetal placental villi (arrow), submucosal oedema and hyperaemic blood vessels. HE. $\times 4 .($ C) Placental villi with inflammatory infiltrate and some detached placental epithelial cells (arrowed). HE. $\times 40$. (D) Labelling of Brucella antigen within inflammatory cells invading the placental villi (example arrowed). IHC. $\times$ 40. (E) Vegetative nodule in the mitral valve (white arrow) of the heart and hyperaemic blood vessels in the dorsal region of the valve (black arrow). (F) Microscopical appearance of the mitral valve shown in (E) with bacterial colonies (arrow) and fibrin deposition HE, $\times 40$. (G) Section from the mitral valve shown in (E) demonstrating Brucella antigen (arrow) and labelled bacterial aggregates. IHC. $\times 10$. $(\mathrm{H})$ Section from the mitral valve shown in (E) demonstrating Brucella antigen within phagocytic cells and some probable bacterial aggregates (arrow). IHC. $\times 40$.

dystrophic calcification and bacterial colonies surrounded by polymorphonuclear cells were also observed in this area (Fig. $3 \mathrm{~F})$. There was also focal degeneration of myocardial fibres that were surrounded by a mild lymphocytic infiltrate and perivascular oedema. Pericardial fibrosis with infiltration of lymphocytes and plasma cells was also present. Brucella antigen was detected by IHC associated with the infiltrating inflammatory cells and the bacterial colonies (Fig. 3G, H).

The results of the present study suggest that the observed stranding of striped dolphins may be directly 
associated with meningoencephalomyelitis caused by infection with $B$. ceti. Although similar pathological changes have been observed in man and in other dolphin species infected with Brucella spp. (Foster et al., 2002; Pappas et al., 2005), these changes are seldom recorded in terrestrial hosts such as cattle, goats, sheep or pigs. In these hosts the main symptoms are related to abortion, placental retention, interstitial mastitis, epididymitis and, in some cases, hygromas (Hagemoser et al., 1988; Meador et al., 1989; Musa et al., 1990). It is notable that neurological or cardiac diseases associated with Brucella are not documented in these domestic animals. The microscopical lesions caused by $B$. ceti were strikingly different from encephalitis caused by morbillivirus, trematode parasites or Toxoplasma, all infections reported in S. coeruleoalba (O'Shea et al., 1991; Domingo et al., 1992; Dubey et al., 2007). In fact, the neuropathology recorded was similar to that described in meningoencephalomyelitis associated with Brucella infection in man (Shakir et al., 1987; Vinod et al., 2007).

Although $B$. ceti was isolated from many of the affected dolphins and Brucella antigen was detected by immunofluorescence in the brain and cerebrospinal fluid, it was not possible to detect Brucella antigen in the central nervous system by IHC. Brucellosis has been diagnosed by immunohistochemical labelling of the brain of one infected dolphin (González et al., 2002), but the lower sensitivity of this technique for identifying Brucella antigens in tissues is recognized (Seidel et al., 2003).

The lesions observed in the heart, liver, lungs, joints and placenta of animals in the presents study suggest that $B$. ceti has the ability to cause chronic infection of multiple organs before it crosses the blood-brain barrier. Similarly, mitral valve lesions have been reported in chronic brucellosis of man (Gon-Je and Song, 2008). The placentitis observed in one dolphin was similar to that reported in two previous cases (Miller et al., 1999); however, despite the placental lesions no significant pathological changes were detected in the fetus. This is noteworthy, as severe placentitis in bovine brucellosis is associated with abortion and these fetuses display severe central nervous system and pulmonary pathology with significant inflammation (Hong et al., 1991).

Descriptions of pathological findings due to natural brucellosis in secondary accidental hosts such as man are sparse (Hunt and Bothwell, 1967; Pappas et al., 2005). Therefore, the severity of the disease observed in the striped dolphins reported here may serve to increase understanding of the natural course of brucellosis in both man and animals.

\section{Conflict of Interest}

The authors do not declare any conflict of interest.

\section{Acknowledgments}

The authors thank G. Hidalgo (CCSS, Costa Rica) for technical assistance with the IHC and M. Domingo (CReSA, Spain) for collaboration with the morbillivirus detection assays and Fundación KETO. This work was funded by the following grants: FIDA-2006-Universidad Nacional, FS-CONARE-UNA/UCR, Florida Ice \& Farm, FORINVES-FV-001-07-MICIT/CONICIT, Ne Tropica 8-N-2005.

\section{References}

Barquero-Calvo E, Chaves-Olarte E, Weiss DS, GuzmánVerri C, Chacón-Díaz G et al. (2007) Brucella abortus uses a stealthy strategy to avoid activation of the innate immune system during the onset of infection. PLoS One, 2, e631. doi:10.1371/journal.pone.0000631.

Baucheron S, Grayon M, Zygmunt MS, Cloeckaert A (2002) Lipopolysaccharide heterogeneity in Brucella strains isolated from marine mammals. Research Microbiology, 153, 277-280.

Boenish T (2001) Immunochemical Staining Methods, 3rd Edit., Dako Cytomation, Carpintería. pp. 1-65.

Brew SD, Perrett LL, Stack JA, MacMillan AP (1999) Human exposure to Brucella recovered from a sea mammal. Veterinary Record, 144, 483.

Dagleish MP, Barley J, Howie FE, Reid RJ, Herman J et al. (2007) Isolation of Brucella species from a diseased atlanto-occipital joint of an Atlantic white-sided dolphin (Lagenorhynchus acutus). Veterinary Record, 160, 876-878.

Domingo M, Visa J, Pumarola M, Marco AJ, Ferrer L et al. (1992) Pathologic and immunohistochemical studies of morbillivirus infection in striped dolphins (Stenella coeruleoalba). Veterinary Pathology, 29, 1-10.

Dubey JP, Morales JA, Sundar N, Velmurugan GV, González-Barrientos CR et al. (2007) Isolation and genetic characterization of Toxoplasma gondii from striped dolphin (Stenella coeruleoalba) from Costa Rica. Journal of Parasitology, 93, 710-711.

Ewalt DR, Payeur JB, Martin BM, Cummins DR, Miller WG (1994) Characterization of a Brucella species from a bottlenose dolphin (Tursiops truncatus). Fournal of Veterinary Diagnostic Investigation, 6, 448-452.

Foster G, MacMillan AP, Godfroid J, Howie F, Ross HM et al. (2002) A review of Brucella spp. infection of sea mammals with particular emphasis on insolates from Scotland. Veterinary Microbiology, 90, 563-580.

Je HG, Song H (2008) Brucella endocarditis in a non-endemic country: first reported case in East Asia. Circulation fournal, 72, 500-501.

González L, Patterson IA, Reid RJ, Foster G, Barberán M et al. (2002) Chronic meningoencephalitis associated with Brucella sp. infection in live-stranded striped dolphins (Stenella coeruleoalba). Journal of Comparative Pathology, 126, 147-152.

Groussaud P, Shankster SJ, Koylass MS, Whatmore AM (2007) Molecular typing divides marine mammal strains of Brucella into at least three groups with distinct 
host preferences. Fournal of Medical Microbiology, 56, 1512-1518.

Hagemoser WA, Deyoe BL, Meador VP (1988) Histopathologic findings in Brucella abortus-infected, pregnant goats. American Fournal of Veterinary Research, 49 274-280.

Hernández-Mora G, González-Barrientos R, Morales J-A, Chaves-Olarte E, Guzmán-Verri C et al. (2008) Neurobrucellosis in stranded dolphins, Costa Rica. Emerging Infectious Diseases, 14, 1430-1433.

Hernández-Mora G, Manire CA, González-Barrientos R, Barquero-Calvo E, Guzmán-Verri C et al. (2009) Serological diagnosis of Brucella infections in odontocetes. Clinical and Vaccine Immunology, 16, 906-915.

Hong CB, Donahue JM Jr., Giles CR, Poonacha KB, Tuttle PA et al. (1991) Brucella abortus-associated meningitis in aborted bovine fetuses. Veterinary Pathology, 28, 492-496.

Hunt AC, Bothwell PW (1967) Histological findings in human brucellosis. Fournal of Clinical Pathology, 20, $267-272$.

Kiernan JA (2003) Histological and Histochemical Methods: Theory and Practice. Oxford University Press, New York. pp. 1-477.

McDonald WL, Jamaludin R, Mackereth G, Hansen M, Humphrey S et al. (2006) Characterization of a Brucella sp. strain as a marine-mammal type despite isolation from a patient with spinal osteomyelitis in New Zealand. Journal of Clinical Microbiology, 44, 4363-4370.

Meador VP, Deyoe BL, Cheville NF (1989) Pathogenesis of Brucella abortus infection of the mammary gland and supramammary lymph node of the goat. Veterinary Pathology, 26, 357-368.

Miller WG, Adams LC, Ficht TA, Cheville NF, Payeur JP et al. (1999) Brucella-induced abortions and infection in bottlenose dolphins (Tursiops truncatus). Fournal of Zoo and Wildlife Medicine, 30, 100-110.

Muñoz PM, García-Castrillo G, López-García P, González-Cueli JC, Barberán M et al. (2006) Isolation of Brucella species from a live-stranded striped dolphin
(Stenella coeruleoalba) in Spain. Veterinary Record, $\mathbf{1 5 8}$ 450-451.

Musa MT, Jahans KL, Fadalla ME (1990) Clinical manifestations of brucellosis in cattle of the southern Darfur Province, western Sudan. Fournal of Comparative Pathology, 103, 95-99.

O'Shea TJ, Homer BL, Greiner EC, Layton AW (1991) Nasitrema sp.-associated encephalitis in a striped dolphin (Stenella coeruleoalba) stranded in the Gulf of Mexico. Journal of Wildlife Diseases, 27, 706-709.

Pappas G, Akritidis N, Bosilkovski M, Tsianos E (2005) Brucellosis. New England Fournal of Medicine, 352, 2325-2336.

Rhyan JC, Gidlewski T, Ewalt DR, Hennager SG, Lambourne DM et al. (2001) Seroconversion and abortion in cattle experimentally infected with Brucella sp. isolated from a Pacific harbor seal (Phoca vitulina richardsi. Fournal of Veterinary Diagnostic Investigation, 13, 379-382.

Seidel G, Pardo CA, Newman-Toker D, Olivi A, Eberhart G (2003) Neurobrucellosis presenting as leukoencephalopathy: the role of cytotoxic $\mathrm{T}$ lymphocytes. Archives of Pathology and Laboratory Medicine, 127, e374-e377.

Shakir RA, Al-din ASN, Araj GF, Lulu AR, Mousa AR et al. (1987) Clinical categories of neurobrucellosis a report on 19 cases. Brain, 110, 213-223.

Sohn AH, Probert WS, Glaser CA, Gupta N, Bollen AW et al. (2003) Human neurobrucellosis with intracerebral granuloma caused by a marine mammal Brucella spp. Emerging Infectious Diseases, 9, 485-488.

Vinod P, Singh MK, Garg MK, Agarwal A (2007) Extensive meningoencephalitis, retrobulbar neuritis, and pulmonary involvement in a patient with neurobrucellosis. Neurology India, 55, 157-159.

$$
\left[\begin{array}{l}
\text { Received, April 10th, } 2009 \\
\text { Accepted, October 14th, } 2009
\end{array}\right]
$$

